REGULAR ARTICLE

\title{
STUDIES ON GENETIC DIVERGENCE AND REACTION TO IRON CHLOROSIS IN AEROBIC RICE ON VERTISOLS IN MAHARASTRA
}

\section{RAJEEV DHAKAL ${ }^{1}$, RITESH KUMAR YADAV ${ }^{*}$, DHAN PRASAD PAUDEL ${ }^{2}$, D. B. DEOSARKAR3}

\author{
${ }^{1}$ Local Initiatives for Biodiversity, Research and Development (LI-BIRD), Pokhara, Nepal \\ ${ }^{2}$ Food and Agriculture Organization, Nepal
}

3Vasantarao Naik Marathwada Krishi Vidhyapeeth, Parbhani, India

\begin{abstract}
The present study was carried out to study the genetic parameters for yield, yield contributing, quality and nutritional characters along with reaction of genotypes against iron chlorosis in vertisols. The experiment consisted of 50 promising genotypes along with standard checks viz., Basmati-370, Parag and Avishkar were grown in randomized block design with three replications. The analysis of variance revealed that all the characters under study. Different genotypes showed some degrees of tolerance to iron chlorosis. And, some other characteristics like number of effective tillers per plant, number of filled grains per panicle, number of unfilled grains per panicle, gel consistency, flag leaf area, straw yield, grain yield per plot and grain yield per plant showed high estimates of phenotypic coefficient of variation, high heritability with high genetic advance. All characters which exhibited high estimates of both genotypic and phenotypic variances coupled with high heritability and high genetic advance can be employed for simple selection for crop improvement. Furthermore, variation in such characters are result of additive gene effects and can be explored and studied for potentialities of being used as parents in breeding program for improvement for particular traits.
\end{abstract}

Keywords: Iron chlorosis, Vertisols, Rice, Heritability, Genetic advance, Variance

\section{INTRODUCTION}

Rice crop is a staple food on which more than half of world population depended for consumption and income generation [1]. About 95 per cent of global output of rice is produced and consumed in developing countries as it is most important food crop for 2.89 billion people in Asia, 40 million in Africa and 1.3 million in the Americas [2]. India is considered as self-sufficient in grain production and is second largest exporter of quality rice [3] where cultivation area of rice is estimated about 43.97 million hectares with production of 104.3 million tones and productivity of $2372 \mathrm{~kg} / \mathrm{ha}$. In Maharashtra, rice is grown over a wide area and ranks $13^{\text {th }}$ in rice production in country. Maharashtra is classified into four rice growing region viz., Konkan, Western Maharashtra, Marathwada and Vidarbha region. The highest area under rice crop is in Vidarbha region (7.954 lakh hectares), while the highest productivity is in Konkan region i.e. $2.75 \mathrm{t} / \mathrm{ha}$ (3.83 t/ha for rough rice). Marathwada region is the non-traditional rice growing area in Maharashtra [4].

Iron is a very important element in plant kingdom [5]. This kind of malady is commonly found in heavy clay soil of Maharashtra [6]. The researchers have reported that grain yield of rice is strongly negatively correlated with iron chlorosis reaction and similarly, contribution of majority of yield contributing characters via iron chlorosis reaction is negative to grain yield $[7 ; 8]$. The rice varieties grown in Marathwada region are old and tall varieties which are inferior in the farmers preferred qualities traits. Varieties like Prabhavati, Sugandha, Parag and Aviskar have been developed by the efforts of agriculture university and research station [8]. Yet, the number of robust and promising varietal options to the farmers which are better in both yield and quality characters, is the major shortfall. Hence, this investigation was carried out to discern the variability in rice genotypes for grain yield, yield contributing characters, nutritional and quality characteristics and assess the reaction of genotypes to iron chlorosis which will be instrumental in identifying the genotypes with better yield and quality traits with iron chlorosis tolerance. Furthermore, the identified genotypes with desired traits can be used in prebreeding. This will be ultimately obliging in enhancing the yield, productivity and area of production of rice in Marathwada region of Maharashtra, India.

\section{MATERIALS AND METHODS}

This study was carried out at farm of Department of Agricultural Botany, Vasantrao Naik Marathwada Krishi

\footnotetext{
Received 06 November 2017; Accepted 31 December 2017

*Corresponding Author

Ritesh Kumar Yadav

Local Initiatives for Biodiversity, Research and Development (LI-BIRD), Pokhara, Nepal

Email: ritesh.yadav@libird.org

(C) This article is open access and licensed under the terms of the Creative Commons Attribution License (http://creativecommons.org/licenses/by/4.o/) which permits unrestricted, use, distribution and reproduction in any medium, or format for any purpose, even commercially provided the work is properly cited. Attribution - You must give appropriate credit, provide a link to the license, and indicate if changes were made.
} 
Vidhyapeeth, Parbhani. Parbhani situated above 408-500 m above sea level and lies between $1805^{8}$ ' to 200 2' north latitude and 760 4' to 770 42' east longitude. Soil of experimental site was found deep black having godrainage. The mean maximum temperature of Parbhani varies from $29{ }^{\circ} \mathrm{C}$ to $45{ }^{\circ} \mathrm{C}$ in summer and minimum temperature varies from $11{ }^{\circ} \mathrm{C}$ to $25{ }^{\circ} \mathrm{C}$ in winter season with average rainfall of 800 to $900 \mathrm{~mm}$ received mostly during June to September. The experimental materials consisting 50 promising genotypes (table 1) of rice including three genotypes viz., Parag, Basmati-370 and Avishkar as checks were allocated in randomized block design in Kharif season of year 2013 and each plot assigned to each genotypes was $0.9 \times 3 \mathrm{~m} 2$.

For all the quantitative characters, observations were recorded from five representative and randomly selected plants while quality characters for each genotype were computed from the bulked samples. For recording observations on iron chlorosis, 1 to 9 scale adopted by Singh and Singh [9] was utilized with modification i.e., taking the $1,3,5$, 7and 9 grades as $0,1,2,3$ and 4 respectively. Observation on parameters like, Days to $50 \%$ flowering, plant height, panicle length, number of effective tillers per plant, number of effective tillers per meter length, days to maturity, number of filled grains per panicle, number of unfilled grains per panicle, 1000 grain weight, grain yield per plant, grain yield per plot were recorded and averaged. For calculating flag leaf area, product of length and breadth of leaf was multiplied by leaf area constant 0.818 as suggested by Jadhav et al. [10]. Grain quality characters viz., L: B ratio, relative density, water absorption, gel consistency, volume expansion, amylose content, crude protein content, gelatinization temperature, grain translucency, kernel elongation ration and elongation index were computed from the bulked grains for each genotypes. Elongation ratio was computed as described by Parikh et al. [11]. Crude protein was estimated by Micro-kjeldhal method [12] and gel consistency was observed by method described by
Cagampang et al. [13]. Amylose content was estimated by method described by Juliano [14].

The statistical analysis were performed on data collected of both quantitative and quality characters from genetic parameters of variability, heritability and expected genetic advance. Analysis of variance was performed as suggested by Panse and Sukhatme [15] while both genotypic and phenotypic variance were estimated by suing formulae as per Burton [16]. Heritability (broad sense) was calculated according to the method suggested by Hanson et al. [17]. Expected genetic advance (EGA) was calculated by the method suggested by Johnson et al. [18].

\section{RESULTS AND DISCUSSION}

\section{Analysis of variance}

Analysis of variance was worked out to assess the variability in the rice genotypes for grain yield, yield components and grain quality characters. The observations are shown in table 2 and 3. There was significant variations in between the genotypes in respect of all the characters viz., plant height, days to 50\% flowering, days to maturity, effective tillers per plant, effective tillers per metre length, panicle length, flag leaf area, 1000 grain weight, number of filled grains per panicle, number of unfilled grains per panicle, grain yield per plant, grain yield per plot and straw yield per plot were significant. The differences among the genotypes with respect to physicochemical characteristics of rice grain, viz., elongation ratio, elongation index, L: B ratio, volume expansion, gel consistency, relative density $(\mathrm{g} / \mathrm{ml})$, water absorption per unit weight of rice, protein content and amylose content were also found significant. The results prove that there is a genetical divergence. These findings are in accordance with the earlier reports [19-21], who also observed significant variability for yield and its components and grain quality characters in rice.

Table 1: List of genotypes included in the study

\begin{tabular}{|c|c|c|c|}
\hline S. No. & Genotypes & S. No. & Genotypes \\
\hline 1. & NDR 1143 & 2. & CR 3693-1-1 \\
\hline 3. & OR 2172-7-1 & 4. & CR 3635-1-1 \\
\hline 5 . & RP 5214-18-6-2-1-1-1-B & 6. & PBNR-O3-10 \\
\hline 7. & GK 5022 & 8. & Rewa 1207-15 \\
\hline 9. & PBNR-04-28 & 10. & CR 3632-1-2 \\
\hline 11. & CRR 599-4-1 & 12. & CR 2991-1-2-1-1-1 \\
\hline 13. & R 2212-RF-75 & 14. & CRR 452-23-1-1-1-1 \\
\hline 15. & CRR 596-8-1 & 16. & CR 3692-1-1 \\
\hline 17. & CB 09516 & 18. & TJP 148 \\
\hline 19. & CB 10-504 & 20. & OR 1946-2-1 \\
\hline 21. & MAS 946 (RC 2) & 22. & RP Bio 4918-166-9 \\
\hline 23. & CR 3695-1-1 & 24. & BAU 408-05 \\
\hline 25. & CR 3634-1-3 & 26. & CR 3690-1-1 \\
\hline 27. & CR 3630-1-3 & 28. & CRR 617-B-47-3 \\
\hline 29. & CR 3633-1-3 & 30. & PBNR-03-07 \\
\hline 31. & R-RF-48 & 32. & MAULS-15 \\
\hline 33. & PBNR-08-07 & 34. & CRR 635-3-2 \\
\hline 35 . & BAU 389-02 & 36. & CR 3616-2-1-2-1-1 \\
\hline 37. & PAU 3832-79-4-3-1 & 38. & RP 5216-13-7-2-1-1-1 \\
\hline 39. & R $1700-2240-4-2295-1$ & 40. & CRR 499-11-2-12-1 \\
\hline 41. & UPRI 2012-16 & 42. & Tulasi \\
\hline 43. & CRR 614-4-1 & 44. & PBNR-08-04 \\
\hline 45. & RP 5125-17-6-3-2-1 & 46. & CRR 451-15-B-A1 \\
\hline 47. & PBNR-O3-2O & 48. & Basmati 370 (check) \\
\hline 49. & Parag (check) & 50. & Avishkar (check) \\
\hline
\end{tabular}


Table 2: ANOVA for yield and yield contributing characters

\begin{tabular}{lllll}
\hline S. No. & Characters & \multicolumn{2}{l}{ Mean sum of squares } & Error \\
\cline { 3 - 5 } & & Replication & Treatments & 41.05 \\
\hline 1. & Days to 50\% flowering & 0.04 & $137.55^{* *}$ & 54.93 \\
2. & Plant height (cm) & 34.60 & $646.06^{* *}$ & 3.06 \\
3. & Panicle length (cm) & 0.01 & $8.94^{* *}$ & 0.15 \\
4. & No. of tillers/plant & 0.07 & $5.36^{* *}$ & 52.42 \\
5. & No. of tillers/meter & 2.36 & $2316.32^{* *}$ & 4.98 \\
6. & Flag Leaf Area (cm $\left.{ }^{2}\right)$ & 11.69 & $121.96^{* *}$ & 61.32 \\
7. & Days to maturity & 0.52 & $173.43^{* *}$ & 98.03 \\
8. & No. of filled grains/panicle & 6.61 & $1547.45^{* *}$ & 16.18 \\
9. & No. of unfilled grains/panicle & 25.01 & $410.35^{* *}$ & 1.18 \\
10. & Test weight (g) & 2.49 & $44.19^{* *}$ & 2.83 \\
11. & Grain yield/plant & 0.76 & $120.72^{* *}$ & 0.01 \\
12. & Grain yield/plot (kg) & 0.005 & $0.20^{* *}$ & 0.01 \\
13. & Straw yield/Plot (kg) & 0.01 & $0.63^{* *}$ & \\
\hline
\end{tabular}

${ }^{* *}$ Significant at $1 \%$ level

Table 3: ANOVA for grain quality characters

\begin{tabular}{|c|c|c|c|c|}
\hline \multirow{2}{*}{$\begin{array}{l}\text { S. } \\
\text { No. }\end{array}$} & \multirow[t]{2}{*}{ Characters } & \multicolumn{3}{|c|}{ Mean sum of squares } \\
\hline & & Replication & Treatments & Error \\
\hline 1. & Elongation ratio & 0.0012 & $0.057^{* *}$ & 0.003 \\
\hline 2. & Elongation index & 0.0002 & $0.019^{* *}$ & 0.006 \\
\hline 3. & Length breadth ratio & 0.018 & $1.052^{* *}$ & 0.023 \\
\hline 4. & Volume expansion & 0.0008 & $1.607^{* *}$ & 0.018 \\
\hline 5. & Water absorption & 0.0021 & $1.026^{* *}$ & 0.018 \\
\hline 6. & Relative density (gm/ml) & 0.0022 & $0.241^{* *}$ & 0.007 \\
\hline 7. & Protein content (\%) & 0.6378 & $7.563^{* *}$ & 0.610 \\
\hline 8. & Gel consistency (mm) & 46.512 & $670.24^{* *}$ & 10.18 \\
\hline 9. & Amylose content (\%) & 0.0096 & $40.566^{* *}$ & 2.265 \\
\hline
\end{tabular}

${ }^{* *}$ Significant at $1 \%$ level

\section{Mean performance}

Mean performance of genotypes for different characters are depicted in table 4, 5, and 6. The genotype PBNR 03-07 was tall in height and had highest flag leaf area. The genotype CR 3690-1-1 had longest panicle length, whereas, genotype PBNR 04-28 had recorded maximum number of effective tillers per plant and number of effective tillers per meter length. The variety Tulasi recorded early in maturity and minimum number of unfilled grains per panicle. The maximum number of filled grains per panicle was recorded in check Avishkar. The genotype RP Bio 4918-166-9 was found to record lowest test weight whereas, genotype CR 452-23-1-1-1-1 recorded minimum straw yield per plot. However, maximum grain yield per plot was recorded by genotype NDR 1143. Among the grain quality characters, genotype CRR 596-8-1 recorded high elongation ratio along with highest elongation index and absorbed significantly more water. The genotype Tulasi has recorded lowest volume expansion ratio. None of the genotype was observed with bold and round grains. Relative density was highest in genotype CRR 617-b-47-3, whereas, genotype PAU 3832-794-3-1 recorded highest protein content. Important grain quality parameters were recorded in genotypes PBNR 0307, PBNR 03-20, PBNR 04-28, PBNR 08-04, CRR 596-8-1, CR 3690-1-1, MAULS-15,R 1700-2240-4-2295-1, and UPRI 499-11-2-12-1. These results are in conformity with findings of Das et al. [22] and Binod et al. [23].

\section{Genotypic and phenotypic coefficient of variation}

The phenotypic coefficient of variation was higher than that of genotypic coefficient of variation. Similar findings were reported by Panwar and Mathur [24]. But the differences between PCV and GCV for many traits were less, suggesting the less influence of environment for traits like plant height, number of filled grains per panicle, 1000 grain weight and grain yield per plant. These results were confirmatory with Devi et al. [25], Prajapati et al. [26], Tuwar et al. [27]. High values of genotypic and phenotypic coefficient of variation were recorded for grain yield per plant (54.32 and 52.46) and number of unfilled grains per panicle (52.78 and 49.80). Similarly, characters like grain yield per plot (36.61 and 36.72) and straw yield (33.88 and 32.84) also showed high GCV and PCV. This result was in accordance with findings of Deosarkar et al. [7] and Fukrei et al. [28]. Grain quality characters viz. (43.73 and 43.01), water absorption (35.49 and 34.57), relative density (24.66 and 23.46) and gel consistency (23.44 and 22.91) were with higher GVC and PCV. Similar finding was reported by Dhanwani et al. [20].

Moderate PCV and GCV were recorded for characters protein content (19.66 and 17.49), test weight (19.64 and 18.87), L: B ratio (19.32 and 18.70), plant height (21.44 and 18.96), amylose content (15.63 and 14.40), elongation ratio (11.45 and 10.48) and elongation index (11.11 and 6.96). Similar results were observed by Dhanwani et al. [20] for L: B ratio (15.63 and 14.40), elongation ratio and elongation index, Subbaiah et al. [29] observed for test weight, elongation ratio and L: B ratio and Kundu et al. [30] reported for test weight. Wide range of genotypic variation was observed in number of effective tillers per plant and grain yield per plant which indicates the predominance of additive gene effects. These results are in agreement with earlier results reported by Shukla et al. [31], Kaul and Kumar [32], Debchoudhary and Das [33] and Chauhan [34]. However, low estimates for GCV and 
PCV were recorded for days to 50\% flowering (9.59 and $3.36)$ followed by panicle length (9.48 and 5.92) and days to maturity (8.00 and 4.92). Low GCV and PCV values for these traits were also reported by Shivani and Reddy [35], Devi et al. [25], Prajapati et al. [26], Annandrao et al. [36] and Singh et al. [37]. Low value for days to 50\% flowering and panicle length have also been reported by Sinha et al. [38] and Sadeghi [39] days to 50\% flowering. Tuwar et al. [27] also observed similar result for days to maturity.

Table 4: Mean values of genotypes for grain yield and yield contributing characters

\begin{tabular}{|c|c|c|c|c|c|c|c|c|}
\hline S. No. & Genotypes & $\begin{array}{l}\text { Days to } \\
50 \% \\
\text { flowering } \\
\end{array}$ & $\begin{array}{l}\text { Plant } \\
\text { height } \\
\text { (cm) }\end{array}$ & $\begin{array}{l}\text { Panicle } \\
\text { length } \\
(\mathrm{cm}) \\
\end{array}$ & $\begin{array}{l}\text { No. of } \\
\text { tillers/plant }\end{array}$ & $\begin{array}{l}\text { No. of } \\
\text { tillers/meter }\end{array}$ & $\begin{array}{l}\text { Flag leaf } \\
\text { area } \\
\left(\mathrm{cm}^{2}\right)\end{array}$ & $\begin{array}{l}\text { Days to } \\
\text { maturity }\end{array}$ \\
\hline 1. & NDR 1143 & 86.33 & 77.86 & 23.33 & 4.80 & 120.23 & 26.29 & 121.66 \\
\hline 2. & CR 3693-1-1 & 89.33 & 96.03 & 21.90 & 5.93 & 112.00 & 38.25 & 122.66 \\
\hline 3. & OR 2172-7-1 & 93.00 & 73.66 & 23.86 & 3.20 & 83.66 & 32.04 & 128.66 \\
\hline 4. & CR 3635-1-1 & 91.66 & 68.93 & 23.46 & 3.26 & 77.00 & 22.43 & 126.00 \\
\hline 5. & $\begin{array}{l}\text { RP 5214-18- } \\
6-2-1-1-1-B\end{array}$ & $104.33^{* * *}$ & 68.23 & 25.76 & 4.13 & 101.33 & $35 \cdot 34$ & $142.33^{* *}$ \\
\hline 6. & PBNR-O3-10 & 83.33 & 94.00 & 26.83 & $7 \cdot 33^{* *}$ & $182.00^{* *}$ & 37.29 & 119.33 \\
\hline 7. & GK 5022 & 96.00 & 52.46 & 21.96 & 4.86 & 118.00 & 32.17 & 132.33 \\
\hline 8. & Rewa 1207-15 & 91.00 & 65.20 & 22.90 & 4.13 & 102.33 & 28.70 & $125 \cdot 33$ \\
\hline 9. & PBNR-04-28 & 86.66 & 78.30 & 21.60 & $8.40^{* * *}$ & $198.66^{* *}$ & 34.58 & 124.33 \\
\hline 10. & CR 3632-1-2 & 95.00 & 65.00 & 23.80 & 5.26 & 126.33 & 26.46 & 130.33 \\
\hline 11. & CRR 599-4-1 & 85.00 & 93.40 & 23.80 & 5.13 & 123.00 & 30.16 & 122.00 \\
\hline 12. & $\begin{array}{l}\text { CR 2991-1-2- } \\
1-1-1\end{array}$ & $101.66^{* *}$ & 68.36 & 25.80 & 3.13 & 80.33 & 31.87 & $138.66^{*}$ \\
\hline 13. & $\begin{array}{l}\text { R 2212-RF- } \\
75\end{array}$ & 85.00 & 91.86 & 24.23 & 3.13 & 91.66 & 26.32 & 120.33 \\
\hline 14. & $\begin{array}{l}\text { CRR 452-23- } \\
1-1-1-1\end{array}$ & 90.66 & 71.26 & 22.65 & $5 \cdot 73$ & 105.66 & 24.37 & 124.66 \\
\hline 15 . & CRR 596-8-1 & 80.00 & 82.33 & 23.56 & $5 \cdot 93$ & 118.00 & 27.10 & 116.33 \\
\hline 16. & CR 3692-1-1 & 94.33 & 74.60 & 22.87 & 4.06 & $103 \cdot 33$ & 31.86 & 130.33 \\
\hline 17. & CB o9 516 & 94.33 & 61.33 & 24.53 & 3.93 & 99.33 & 32.17 & 129.33 \\
\hline 18. & TJP 148 & 81.00 & 76.73 & 23.91 & 5.60 & 140.33 & 37.51 & $115 \cdot 33$ \\
\hline 19. & CB 10-504 & $95 \cdot 33$ & 66.13 & 21.30 & 4.66 & 114.00 & 30.78 & 131.00 \\
\hline 20. & OR 1946-2-1 & $99.66^{*}$ & 61.60 & 22.54 & 3.93 & 93.00 & 28.76 & $136.33^{*}$ \\
\hline 21. & $\begin{array}{l}\text { MAS } 946 \text { (RC } \\
2)\end{array}$ & $98.33^{*}$ & 50.80 & 21.57 & 5.20 & 115.66 & 26.38 & $135.33^{*}$ \\
\hline 22. & $\begin{array}{l}\text { RP Bio 4918- } \\
166-9\end{array}$ & 94.66 & 58.86 & 22.18 & 4.26 & 107.00 & 33.67 & 128.66 \\
\hline 23 . & CR 3695-1-1 & 91.33 & 56.53 & 22.66 & 3.93 & 104.00 & 20.54 & 126.33 \\
\hline 24. & BAU 408-05 & 79.00 & 84.46 & 21.75 & 5.26 & 127.66 & 20.96 & 111.66 \\
\hline 25 . & CR 3634-1-3 & 96.66 & 64.83 & 22.76 & 5.20 & 117.00 & 24.63 & 129.66 \\
\hline 26. & CR 3690-1-1 & 97.66 & 59.13 & 28.90 & 6.13 & 112.00 & 22.35 & $133 \cdot 33$ \\
\hline 27. & CR 3630-1-3 & 92.66 & 66.30 & 23.91 & 4.80 & 121.66 & 28.48 & 126.00 \\
\hline 28 . & $\begin{array}{l}\text { CRR 617-B- } \\
47-3\end{array}$ & 81.00 & 83.13 & 23.45 & 4.73 & 115.00 & 21.56 & 114.66 \\
\hline 29. & CR 3633-1-3 & 88.00 & 87.00 & 22.65 & 4.60 & 108.33 & 22.42 & 123.00 \\
\hline 30. & PBNR-03-07 & 84.66 & $111.56^{* *}$ & 27.02 & $8.00^{* *}$ & $184.00^{* * *}$ & $45 \cdot 91^{* *}$ & 122.00 \\
\hline 31. & $\mathrm{R}-\mathrm{RF}-48$ & 93.66 & 53.20 & 20.28 & 4.06 & 93.33 & $19 \cdot 32$ & 131.66 \\
\hline 32. & MAULS-15 & 78.33 & 85.80 & 23.93 & 5.80 & 135.00 & 30.15 & 111.66 \\
\hline 33. & PBNR-08-07 & 85.00 & 93.43 & 24.83 & 6.53 & 156.33 & 40.07 & 120.33 \\
\hline 34. & CRR 635-3-2 & 84.66 & 94.06 & $24 \cdot 33$ & 5.60 & 127.66 & $25 \cdot 32$ & 115.66 \\
\hline 35. & BAU 389-02 & 89.66 & 72.96 & 24.10 & 3.53 & 97.00 & 21.00 & 124.66 \\
\hline 36. & $\begin{array}{l}\text { CR 3616-2-1- } \\
2-1-1\end{array}$ & 91.66 & 80.33 & 24.23 & $3 \cdot 53$ & 92.33 & 29.49 & 126.00 \\
\hline 37. & $\begin{array}{l}\text { PAU 3832- } \\
79-4-3-1\end{array}$ & 90.00 & 50.63 & 24.47 & 4.13 & 101.66 & 25.41 & 126.00 \\
\hline 38. & $\begin{array}{l}\text { RP 5216-13- } \\
7-2-1-1-1\end{array}$ & 93.33 & 53.03 & 23.96 & 4.06 & 104.33 & 31.44 & 129.66 \\
\hline 39. & $\begin{array}{l}\text { R 1700- } \\
2240-4^{-} \\
2295^{-1}\end{array}$ & $99.00^{*}$ & 46.33 & 22.26 & 3.26 & 113.66 & 22.82 & $136.00^{*}$ \\
\hline 40. & $\begin{array}{l}\text { CRR 499-11- } \\
2-12-1\end{array}$ & 88.66 & 75.00 & 20.50 & 4.13 & 109.33 & 27.46 & 124.33 \\
\hline 41. & $\begin{array}{l}\text { UPRI 2012- } \\
16\end{array}$ & 91.66 & 71.20 & 22.20 & 2.90 & 87.33 & 28.17 & 127.33 \\
\hline 42. & Tulasi & 79.66 & 53.26 & 21.56 & 4.60 & 116.33 & 19.90 & 108.00 \\
\hline
\end{tabular}




\begin{tabular}{|c|c|c|c|c|c|c|c|c|}
\hline 43. & CRR 614-4-1 & 81.00 & 89.66 & 25.66 & $7.13^{* * *}$ & 171.33 & 23.42 & 114.00 \\
\hline 44. & $\begin{array}{l}\text { PBNR-08- } \\
\text { O4 }\end{array}$ & 81.33 & 82.30 & $25 \cdot 50$ & $6.93^{* * *}$ & 164.00 & $44.06^{* *}$ & 116.33 \\
\hline 45 . & $\begin{array}{l}\text { RP 5125-17- } \\
6-3-2-1\end{array}$ & 89.33 & 73.13 & 22.36 & 3.66 & 102.00 & 28.31 & 119.66 \\
\hline 46. & $\begin{array}{l}\text { CRR 451-15- } \\
\text { B-A1 }\end{array}$ & 78.00 & $73 \cdot 33$ & 22.90 & 3.60 & 104.33 & 27.97 & 114.00 \\
\hline 47. & PBNR-O3-20 & 91.00 & 87.00 & 24.60 & $7.53^{* *}$ & 159.66 & 38.78 & 119.33 \\
\hline 48. & $\begin{array}{l}\text { Basmati-370 } \\
\text { (ch) }\end{array}$ & 87.33 & 92.26 & 26.73 & 5.00 & 127.66 & 29.18 & 122.33 \\
\hline 49. & Parag (ch) & 78.66 & 78.80 & 24.36 & 4.93 & 166.66 & 38.53 & $115 \cdot 33$ \\
\hline \multirow[t]{5}{*}{50.} & Avishkar (ch) & 79.66 & 85.40 & 24.69 & 5.93 & 134.00 & 37.01 & 117.00 \\
\hline & Mean & 89.17 & 74.022 & 23.62 & 4.91 & 119.33 & 29.38 & 124.14 \\
\hline & SE & 3.69 & 4.279 & 1.01 & 0.22 & 4.18 & 1.28 & $4.5^{2}$ \\
\hline & CD 5\% & 10.38 & 12.008 & 2.84 & 0.64 & 11.73 & 3.61 & 12.69 \\
\hline & CV (\%) & 7.18 & 10.012 & 7.41 & 8.04 & 6.06 & 7.59 & 6.30 \\
\hline
\end{tabular}

* and ${ }^{* *}$ indicates significant at 5 and 1 per cent level, respectively

Table 5: Mean values of genotypes for grain yield and yield contributing characters

\begin{tabular}{|c|c|c|c|c|c|c|c|c|}
\hline S. No. & Genotypes & $\begin{array}{l}\text { Filled } \\
\text { grains } \\
\text { per } \\
\text { panicle }\end{array}$ & $\begin{array}{l}\text { Unfilled } \\
\text { grains per } \\
\text { panicle }\end{array}$ & $\begin{array}{l}\text { Test } \\
\text { weight } \\
\text { (g) }\end{array}$ & $\begin{array}{l}\text { Grain } \\
\text { yield per } \\
\text { plant(g) }\end{array}$ & $\begin{array}{l}\text { Grain } \\
\text { yield/plot } \\
\text { (g) }\end{array}$ & $\begin{array}{l}\text { Straw } \\
\text { yield/plot } \\
\text { (g) }\end{array}$ & $\begin{array}{l}\text { Iron } \\
\text { chlorosis } \\
\text { reaction }\end{array}$ \\
\hline 1. & NDR 1143 & 132.56 & 22.10 & 18.73 & 11.80 & $1.418^{*}$ & 1.788 & 2 \\
\hline 2. & CR 3693-1-1 & 101.43 & 21.60 & 21.66 & 13.59 & 0.676 & 1.207 & 3 \\
\hline 3. & OR 2172-7-1 & 107.70 & 14.06 & 23.40 & 8.28 & 0.402 & 1.049 & 4 \\
\hline 4. & CR 3635-1-1 & 103.80 & 17.50 & 22.13 & 7.57 & 0.837 & 1.150 & 3 \\
\hline 5. & $\begin{array}{l}\text { RP 5214-18-6-2- } \\
1-1-1-B\end{array}$ & 102.70 & 40.33 & 13.53 & 5.83 & 0.463 & 1.251 & 4 \\
\hline 6. & PBNR-O3-10 & 134.10 & 31.56 & 24.53 & 24.25 & 1.114 & 2.477 & 0 \\
\hline 7. & GK 5022 & 101.56 & 36.26 & 14.80 & 7.250 & 0.377 & 1.406 & 1 \\
\hline 8. & Rewa 1207-15 & 105.40 & 20.70 & 18.93 & 8.283 & 0.736 & 1.193 & 3 \\
\hline 9. & PBNR-O4-28 & 153.80 & 14.80 & 17.90 & 23.04 & 0.694 & 2.083 & o \\
\hline 10. & CR 3632-1-2 & 96.70 & 23.66 & 17.40 & 8.72 & 0.633 & 1.107 & 3 \\
\hline 11. & CRR 599-4-1 & 125.96 & 21.80 & 17.70 & 11.51 & 0.518 & 0.984 & 2 \\
\hline 12. & $\begin{array}{l}\text { CR 2991-1-2-1-1- } \\
1\end{array}$ & 97.66 & 11.66 & 20.93 & 6.33 & 0.510 & 1.291 & 4 \\
\hline 13. & R 2212-RF-75 & 151.10 & 21.00 & 20.80 & 9.61 & 0.928 & 1.598 & 1 \\
\hline 14. & $\begin{array}{l}\text { CRR 452-23-1-1- } \\
1-1\end{array}$ & 109.40 & 20.13 & 18.80 & 11.70 & 0.494 & 0.598 & 4 \\
\hline 15 . & CRR 596-8-1 & 129.30 & 17.93 & 19.66 & 15.04 & 0.502 & 0.967 & 1 \\
\hline 16. & CR 3692-1-1 & 108.93 & 20.74 & 21.20 & 9.38 & 0.887 & 1.344 & 2 \\
\hline 17. & CB 09516 & 96.10 & 30.86 & 14.33 & $5 \cdot 33$ & 0.439 & 1.031 & 4 \\
\hline 18. & TJP 148 & 125.43 & 16.73 & 23.33 & 16.51 & 0.849 & 1.760 & o \\
\hline 19. & CB 10-504 & 153.76 & $77 \cdot 30^{* *}$ & 15.10 & 10.90 & 0.742 & 1.460 & 2 \\
\hline 20. & OR 1946-2-1 & 133.03 & 36.70 & 13.26 & 7.03 & 0.376 & 0.974 & 4 \\
\hline 21. & MAS 946 (RC 2) & 85.96 & 17.03 & 20.20 & 9.15 & 0.624 & 1.318 & 2 \\
\hline 22. & $\begin{array}{l}\text { RP Bio 4918- } \\
166-9\end{array}$ & 95.80 & 30.46 & 12.46 & 5.18 & 0.413 & 1.273 & 4 \\
\hline 23. & CR 3695-1-1 & 99.10 & 25.16 & 21.40 & 8.29 & 0.623 & 1.092 & 2 \\
\hline 24. & BAU 408-05 & 136.70 & 17.60 & 18.46 & 13.21 & 0.855 & 1.112 & 1 \\
\hline 25 . & CR 3634-1-3 & 122.70 & 15.96 & 15.46 & 9.75 & 0.956 & 1.331 & 3 \\
\hline 26. & CR 3690-1-1 & 109.50 & 26.53 & 19.06 & 12.65 & 0.608 & 1.067 & 3 \\
\hline 27. & CR 3630-1-3 & 119.76 & 24.73 & 23.80 & 13.84 & 0.988 & 1.316 & 3 \\
\hline 28. & CRR 617-B-47-3 & 85.53 & 26.60 & 20.86 & 8.49 & 0.544 & 0.998 & 3 \\
\hline 29. & CR 3633-1-3 & 111.90 & 22.90 & 22.00 & 11.38 & 0.986 & 1.367 & 2 \\
\hline 30. & PBNR-03-07 & 151.06 & 19.06 & $25 \cdot 50$ & 29.63 & 0.998 & 2.655 & o \\
\hline 31. & $\mathrm{R}-\mathrm{RF}-48$ & 100.66 & 31.60 & 19.13 & 9.19 & 0.571 & 0.871 & 4 \\
\hline 32. & MAULS-15 & 102.60 & 12.26 & 25.06 & 14.75 & 0.995 & 1.854 & o \\
\hline 33. & PBNR-08-07 & 150.06 & 26.63 & 20.93 & 19.32 & 1.012 & 1.646 & o \\
\hline 34. & CRR 635-3-2 & 99.53 & 35.33 & 18.30 & 10.25 & 0.802 & 1.771 & 2 \\
\hline 35. & BAU 389-02 & 127.60 & 11.13 & 19.86 & 8.85 & 0.727 & 1.483 & 2 \\
\hline 36. & $\begin{array}{l}\text { CR 3616-2-1-2-1- } \\
1\end{array}$ & 117.90 & 17.70 & 21.13 & 8.87 & 0.875 & 1.049 & 3 \\
\hline 37. & PAU 3832-79-4- & 92.56 & 11.76 & 24.93 & 9.43 & 0.511 & 0.857 & 3 \\
\hline
\end{tabular}




\begin{tabular}{|c|c|c|c|c|c|c|c|c|}
\hline 38. & $\begin{array}{l}3-1 \\
\text { RP 5216-13-7-2- } \\
1-1-1\end{array}$ & 81.70 & 18.36 & 13.20 & 4.49 & 0.565 & 1.151 & 3 \\
\hline 39. & $\begin{array}{l}\text { R 1700-2240-4- } \\
2295^{-1}\end{array}$ & 121.50 & 28.83 & 16.86 & 6.71 & 0.618 & 1.452 & 2 \\
\hline 40. & $\begin{array}{l}\text { CRR 499-11-2- } \\
12-1\end{array}$ & 84.20 & 20.00 & 14.60 & 5.17 & 0.586 & 1.320 & 2 \\
\hline 41. & UPRI 2012-16 & 85.86 & 24.16 & 20.06 & 5.02 & 1.005 & 1.167 & 3 \\
\hline 42. & Tulasi & 119.66 & 4.23 & 18.56 & 10.18 & 1.141 & 1.211 & 2 \\
\hline 43. & CRR 614-4-1 & 122.80 & 10.93 & 24.90 & 22.27 & 0.921 & 2.159 & $\mathrm{O}$ \\
\hline 44. & PBNR-08-04 & 127.60 & 17.73 & 24.53 & 22.15 & 1.146 & 1.846 & $\mathrm{O}$ \\
\hline 45 & $\begin{array}{l}\text { RP 5125-17-6-3- } \\
2-1\end{array}$ & 120.63 & 25.20 & 21.60 & 9.40 & 0.777 & 1.081 & 2 \\
\hline 46. & CRR 451-15-B-A1 & 112.53 & 21.20 & 21.00 & 8.24 & 0.502 & 0.670 & 3 \\
\hline 47. & PBNR-O3-20 & 117.26 & 14.93 & $24 \cdot 30$ & 21.32 & $1.299^{*}$ & 1.900 & O \\
\hline 48. & $\begin{array}{l}\text { Basmati-370 } \\
\text { (ch) }\end{array}$ & 69.36 & 51.03 & 20.46 & 7.29 & 0.357 & 1.479 & 2 \\
\hline 49. & Parag (ch) & 154.13 & 11.73 & 28.53 & 21.21 & 1.086 & 2.724 & $\mathrm{O}$ \\
\hline 50. & Avishkar (ch) & 179.23 & 12.46 & 28.53 & 30.18 & 1.052 & 1.601 & $\mathrm{O}$ \\
\hline Mean & & 115.51 & 23.01 & 20.05 & 12.59 & 0.758 & 1.391 & -- \\
\hline $\mathrm{SE} \pm$ & & $5 \cdot 71$ & 2.32 & 0.62 & 0.97 & 0.062 & 0.067 & -- \\
\hline CD 5\% & & 16.04 & 6.51 & 1.76 & 2.72 & 0.174 & 0.188 & -- \\
\hline $\mathrm{CV} \%$ & & 8.57 & 17.48 & 5.42 & 14.09 & 14.25 & 8.37 & -- \\
\hline
\end{tabular}

Table 6: Mean values of genotypes for grain quality characters of rice

\begin{tabular}{|c|c|c|c|c|c|c|c|c|c|c|c|c|c|}
\hline S. No. & Genotypes & ER & EI & $\mathbf{L} / \mathbf{B}$ & VE & WA & RD & $\begin{array}{l}\text { PC } \\
(\%)\end{array}$ & GC & $\begin{array}{l}\mathrm{AC} \\
(\%)\end{array}$ & GT & WB & $\mathbf{A R}$ \\
\hline 1. & NDR 1143 & 1.27 & 0.98 & 2.50 & 1.55 & 1.32 & 0.87 & $10.83^{* *}$ & 78.66 & 22.33 & Intermediate & Absent & Absent \\
\hline 2. & CR 3693-1-1 & 1.29 & 0.96 & 2.57 & 0.67 & 1.53 & 0.94 & $9.99^{*}$ & 73.33 & $27.93^{*}$ & Intermediate & Present & Strong \\
\hline 3. & OR 2172-7-1 & 1.17 & 0.90 & 2.93 & 2.57 & 1.55 & 0.99 & 7.99 & 82.66 & 22.43 & Intermediate & Present & Mild \\
\hline 4. & CR 3635-1-1 & 1.26 & 0.93 & 2.98 & 1.68 & $2.52^{* * *}$ & 1.06 & 8.67 & 78.66 & $27.96^{*}$ & Intermediate & Present & Mild \\
\hline 5. & $\begin{array}{l}\text { RP 5214-18- } \\
6-2-1-1-1-B\end{array}$ & 1.21 & 0.95 & 2.94 & 2.44 & $2.39^{* * *}$ & 1.17 & $10.99^{* *}$ & $65 \cdot 33$ & 23.56 & High & Absent & Mild \\
\hline 6. & $\begin{array}{l}\text { PBNR-O3- } \\
10\end{array}$ & 1.27 & 0.93 & 2.61 & 1.38 & 1.65 & 1.13 & 8.26 & 55.66 & 20.81 & Intermediate & Absent & Mild \\
\hline 7. & GK 5022 & 1.32 & 0.85 & 3.12 & 2.00 & 1.75 & 1.18 & 9.33 & 62.66 & 19.40 & Intermediate & Absent & Strong \\
\hline 8. & $\begin{array}{l}\text { Rewa 1207- } \\
15\end{array}$ & 1.36 & 0.87 & 3.26 & 1.63 & $2.60^{* * *}$ & 0.89 & 7.17 & $75 \cdot 33$ & 22.32 & Intermediate & Absent & Mild \\
\hline 9. & $\begin{array}{l}\text { PBNR-O4- } \\
28\end{array}$ & 1.20 & 0.75 & 3.60 & 0.72 & 1.45 & 1.23 & 6.84 & 64.66 & 24.53 & Intermediate & Absent & Strong \\
\hline 10. & $\begin{array}{l}\mathrm{CR} 3632-1- \\
2\end{array}$ & 1.17 & 0.79 & 3.22 & 1.22 & $2.32^{* *}$ & 0.92 & 8.16 & 75.66 & $25 \cdot 37$ & Intermediate & Absent & Mild \\
\hline 11. & $\begin{array}{l}\text { CRR 599-4- } \\
1\end{array}$ & 1.19 & 0.89 & 2.82 & $2.87^{*}$ & 1.46 & 1.74 & 7.66 & 71.00 & 20.17 & Intermediate & Absent & Mild \\
\hline 12. & $\begin{array}{l}\text { CR 2991-1- } \\
2-1-1-1\end{array}$ & 1.21 & 0.98 & 2.39 & 1.44 & 1.34 & 1.13 & 7.04 & 73.00 & 19.87 & High & Present & Mild \\
\hline 13. & $\begin{array}{l}\mathrm{R} 2212-\mathrm{RF}- \\
75\end{array}$ & 1.16 & 1.04 & 3.10 & 1.80 & $1.83^{*}$ & 1.19 & $10.16^{* *}$ & 45.66 & 21.23 & Intermediate & Present & Strong \\
\hline 14. & $\begin{array}{l}\text { CRR 452- } \\
23-1-1-1-1\end{array}$ & 1.25 & 0.87 & 2.99 & 2.06 & $2.77^{* *}$ & 0.99 & $7 \cdot 35$ & 78.66 & 27.79 & Intermediate & Absent & Strong \\
\hline 15 . & $\begin{array}{l}\text { CRR 596-8- } \\
1\end{array}$ & 1.68 & 1.13 & 3.28 & 2.63 & $2.91^{* *}$ & 1.06 & 7.04 & 58.66 & $25 \cdot 33$ & Intermediate & Present & Mild \\
\hline 16. & CR 3692-1-1 & 1.29 & 0.99 & 2.50 & 1.35 & 1.66 & 1.07 & 8.99 & 65.66 & $30.24^{* * *}$ & Intermediate & Present & Strong \\
\hline 17. & CB o9 516 & 1.19 & 0.99 & 3.16 & 1.85 & 2.59 & 0.94 & $11.32^{* *}$ & 46.66 & $32.51^{* * *}$ & Intermediate & Absent & Mild \\
\hline 18. & TJP 148 & 1.31 & 0.92 & 3.13 & 2.00 & 1.62 & 1.35 & $7 \cdot 33$ & 65.33 & $28.46^{* *}$ & Intermediate & Absent & Strong \\
\hline 19. & CB 10-504 & 1.26 & 0.89 & 2.84 & $2.89^{* *}$ & $2.59^{* * *}$ & 1.36 & $9.83^{*}$ & 62.33 & 27.05 & High & Present & Mild \\
\hline 20. & $\begin{array}{l}\text { OR 1946-2- } \\
1\end{array}$ & 1.15 & 0.87 & 2.77 & 1.44 & 1.22 & 1.27 & $9.63^{*}$ & 74.66 & $30.63^{* * *}$ & High & Absent & Mild \\
\hline 21. & $\begin{array}{l}\text { MAS } 946 \\
(\mathrm{RC} 2)\end{array}$ & 1.15 & 0.81 & 2.93 & 1.28 & $1.91^{*}$ & 0.95 & $10.32^{* *}$ & $54 \cdot 33$ & 23.39 & Intermediate & Absent & Mild \\
\hline 22. & $\begin{array}{l}\text { RP Bio } \\
4918-166-9\end{array}$ & 1.37 & 0.99 & 2.70 & 2.33 & $2.47^{* *}$ & 1.07 & 6.67 & 68.33 & 20.62 & Intermediate & Absent & Mild \\
\hline 23. & CR 3695-1-1 & 1.29 & 0.92 & 3.49 & 2.67 & 1.45 & 1.78 & 7.03 & 28.33 & $29.86^{* *}$ & Intermediate & Present & Mild \\
\hline 24. & $\begin{array}{l}\text { BAU 408- } \\
\text { O5 }\end{array}$ & 1.28 & 0.96 & 2.80 & 0.93 & 1.22 & 1.08 & $10.16^{* *}$ & 59.33 & 21.64 & Intermediate & Absent & Mild \\
\hline 25 . & $\begin{array}{l}\text { CR 3634-1- } \\
3\end{array}$ & 1.17 & 0.90 & 3.06 & 0.67 & 1.07 & 0.85 & $7 \cdot 50$ & 74.66 & 26.65 & Intermediate & Absent & Strong \\
\hline 26. & CR 3690-1-1 & 1.44 & 0.96 & 3.68 & 1.56 & $2.61^{* *}$ & 0.93 & 6.83 & $77 \cdot 33$ & 24.62 & Intermediate & Absent & Absent \\
\hline 27. & $\begin{array}{l}\text { CR 3630-1- } \\
3\end{array}$ & 1.32 & 0.90 & 3.04 & 0.53 & 0.78 & 1.10 & $7 \cdot 54$ & 76.33 & 26.73 & Intermediate & Present & Mild \\
\hline
\end{tabular}




\begin{tabular}{|c|c|c|c|c|c|c|c|c|c|c|c|c|c|}
\hline 28. & $\begin{array}{l}\text { CRR 617-B- } \\
47-3\end{array}$ & 1.18 & 0.91 & 3.00 & 1.80 & 0.61 & $1.87^{*}$ & $10.33^{* * *}$ & 26.66 & 25.68 & Intermediate & Absent & Mild \\
\hline 29. & $\begin{array}{l}\text { CR 3633-1- } \\
3\end{array}$ & 1.28 & 0.80 & 2.58 & 2.54 & 1.47 & 1.56 & $10.16^{* *}$ & 71.33 & 18.53 & Intermediate & Present & Mild \\
\hline 30. & $\begin{array}{l}\text { PBNR-03- } \\
\text { O7 }\end{array}$ & 1.41 & 0.80 & 3.15 & 1.00 & 1.22 & 1.19 & 6.54 & 61.33 & $24 \cdot 30$ & Intermediate & Absent & Strong \\
\hline 31. & R-RF-48 & 1.15 & 0.86 & 3.22 & 1.67 & 1.65 & 1.17 & $11.03^{* *}$ & 76.33 & $29.38^{* *}$ & Intermediate & Present & Mild \\
\hline 32. & MAULS-15 & 1.19 & 0.94 & 3.11 & 0.50 & 0.75 & 0.94 & $10.32^{* *}$ & 58.33 & 25.83 & High & Absent & Mild \\
\hline 33. & $\begin{array}{l}\text { PBNR-08- } \\
07\end{array}$ & 1.40 & 0.86 & 3.27 & 1.81 & $1.88^{* *}$ & 1.13 & 7.22 & 44.33 & 19.39 & High & Absent & Mild \\
\hline 34. & $\begin{array}{l}\text { CRR 635-3- } \\
2\end{array}$ & 1.09 & 0.92 & $3 \cdot 37$ & 2.38 & $2.20^{* * *}$ & 1.34 & 7.13 & 52.36 & $30.24^{* *}$ & High & Absent & Strong \\
\hline 35 . & $\begin{array}{l}\text { BAU 389- } \\
\text { O2 }\end{array}$ & 1.09 & 0.86 & 3.20 & 0.62 & 0.52 & 1.26 & $9.99^{*}$ & 96.33 & $27.33^{*}$ & Intermediate & Absent & Absent \\
\hline 36. & $\begin{array}{l}\text { CR 3616-2- } \\
1-2-1-1\end{array}$ & 1.32 & 0.94 & 2.82 & 0.60 & 1.39 & 0.71 & $9.66^{*}$ & 69.66 & 24.33 & High & Present & Mild \\
\hline 37. & $\begin{array}{l}\text { PAU 3832- } \\
79-4-3-1\end{array}$ & 1.22 & 0.90 & 3.15 & 2.60 & 1.42 & 1.74 & $11.82^{* *}$ & 57.66 & $29.50^{* * *}$ & Intermediate & Absent & Strong \\
\hline 38. & $\begin{array}{l}\text { RP 5216-13- } \\
7-2-1-1-1\end{array}$ & 1.15 & 0.88 & 3.52 & $3.00^{* * *}$ & $2.29^{* *}$ & 1.24 & $10.83^{* *}$ & 58.33 & $28.40^{* *}$ & Intermediate & Present & Strong \\
\hline 39. & $\begin{array}{l}\text { R 1700- } \\
2240-4- \\
2295^{-1}\end{array}$ & 1.13 & 0.87 & 3.70 & 2.17 & 1.27 & 1.12 & 7.21 & 52.66 & 20.56 & Intermediate & Absent & Mild \\
\hline 40. & $\begin{array}{l}\text { CRR 499- } \\
11-2-12-1\end{array}$ & 1.17 & 0.90 & 3.15 & 2.00 & $2.16^{* *}$ & 1.25 & 8.31 & 78.33 & 21.99 & High & Absent & Mild \\
\hline 41. & $\begin{array}{l}\text { UPRI 2012- } \\
16\end{array}$ & 1.23 & 1.05 & 2.43 & 2.00 & 1.16 & 1.76 & $11.59^{* *}$ & 88.66 & $29.33^{* *}$ & Intermediate & Present & Mild \\
\hline 42. & Tulasi & 1.62 & 1.07 & $3 \cdot 34$ & 0.33 & 1.05 & 0.57 & $7 \cdot 54$ & 24.33 & 25.63 & Intermediate & Present & Strong \\
\hline 43. & $\begin{array}{l}\text { CRR 614-4- } \\
1\end{array}$ & 1.26 & 0.89 & $3 \cdot 56$ & 0.60 & 1.15 & 0.94 & 7.66 & 83.66 & 19.70 & Intermediate & Absent & Mild \\
\hline 44. & $\begin{array}{l}\text { PBNR-08- } \\
\text { O4 }\end{array}$ & 1.21 & 0.96 & 3.47 & 1.55 & 1.49 & 1.24 & 8.09 & 68.33 & 20.53 & Intermediate & Absent & Mild \\
\hline 45 . & $\begin{array}{l}\text { RP 5125-17- } \\
6-3-2-1\end{array}$ & 1.27 & 0.94 & 2.47 & 1.88 & $2.15^{* *}$ & 1.06 & $11.16^{* *}$ & 73.66 & $29.03^{* *}$ & High & Present & Mild \\
\hline 46. & $\begin{array}{l}\text { CRR 451-15- } \\
\text { B-A1 }\end{array}$ & 1.36 & 0.91 & 3.19 & 1.71 & 1.68 & 1.26 & $7 \cdot 38$ & $54 \cdot 33$ & 25.80 & High & Absent & Strong \\
\hline 47. & $\begin{array}{l}\text { PBNR-O3- } \\
20\end{array}$ & 1.19 & 0.81 & 2.55 & 0.90 & 1.47 & 1.16 & 7.42 & 46.33 & 24.83 & Intermediate & Absent & Strong \\
\hline 48. & $\begin{array}{l}\text { Basmati- } \\
370 \text { (ch) }\end{array}$ & 1.67 & 1.09 & 3.63 & 2.60 & 1.31 & 1.7 & 8.33 & 61.33 & 20.35 & Intermediate & Absent & Strong \\
\hline 49. & Parag (ch) & 1.34 & 0.92 & 3.24 & 1.60 & 1.41 & 1.28 & $7 \cdot 37$ & 72.66 & 21.14 & Intermediate & Absent & Mild \\
\hline \multirow[t]{5}{*}{50.} & $\begin{array}{l}\text { Avishkar } \\
\text { (ch) }\end{array}$ & 1.64 & 1.00 & 4.61 & 2.57 & 1.55 & 1.53 & $7 \cdot 45$ & 65.33 & 24.80 & High & Absent & Strong \\
\hline & Mean & 1.27 & 0.92 & 3.08 & 1.69 & 1.677 & 1.18 & 8.70 & 64.72 & 24.80 & -- & -- & -- \\
\hline & $\mathrm{SE} \pm$ & 0.03 & 0.04 & 0.08 & 0.07 & 0.08 & 0.05 & 0.45 & 1.84 & 0.87 & -- & -- & -- \\
\hline & CD 5\% & 0.09 & 0.13 & 0.24 & 0.22 & 0.22 & 0.14 & 1.26 & 5.17 & 2.44 & -- & -- & -- \\
\hline & CV \% & 4.62 & 8.66 & 4.87 & 7.94 & 8.05 & $7 \cdot 52$ & 8.98 & 4.93 & 6.06 & -- & -- & -- \\
\hline
\end{tabular}

* and ** indicates significant at 5 and 1 per cent level, respectively, ER-elongation ratio, EI-elongation index, L/B-length breadth ratio, WA-water absorption, VE-volume expansion, PC-protein content, RD-relative density, GC-gel consistency, ACamylose content, GT-gelatinization temperature, WB-chalkiness, AR-aroma.

Table 7: Estimates of variability, broad sense heritability and genetic advance for grain yield and yield contributing characters

\begin{tabular}{|c|c|c|c|c|c|c|c|c|c|}
\hline $\begin{array}{l}\text { S. } \\
\text { No. }\end{array}$ & Characteristics & Range & $\operatorname{mean} \pm \mathrm{SE}$ & $\sigma^{2} \mathbf{g}$ & $\boldsymbol{\sigma}^{2} \mathbf{p}$ & GCV & PCV & $\begin{array}{l}\mathbf{h}^{2} \\
\text { (broad } \\
\text { sense) }\end{array}$ & $\begin{array}{l}\text { G. A. as \% } \\
\text { of mean } \\
(5 \%)\end{array}$ \\
\hline 1. & $\begin{array}{l}\text { Days to } 50 \% \\
\text { flowering }\end{array}$ & $\begin{array}{l}78.00- \\
104.33\end{array}$ & $89.17 \pm 3.69$ & 32.165 & 73.220 & 6.360 & 9.596 & 43.9 & 8.64 \\
\hline 2. & Plant height $(\mathrm{cm})$ & $\begin{array}{l}50.63^{-} \\
111.56\end{array}$ & $74.02 \pm 4.28$ & 197.046 & $215 \cdot 977$ & 18.964 & 21.444 & 78.2 & 34.54 \\
\hline 3. & $\begin{array}{l}\text { Panicle Length } \\
(\mathrm{cm})\end{array}$ & $\begin{array}{l}20.28- \\
28.90\end{array}$ & $23.62 \pm 1.01$ & 1.959 & 5.024 & 5.925 & 9.489 & 39.0 & 7.62 \\
\hline 4. & No. of tiller/plant & $2.90-8.40$ & $4.91 \pm 0.22$ & 1.735 & 1.891 & 26.811 & 27.992 & 91.7 & 52.89 \\
\hline 5. & $\begin{array}{l}\text { No. of } \\
\text { tiller/meter }\end{array}$ & $\begin{array}{l}77.00- \\
198.66\end{array}$ & $119.33 \pm 4.18$ & 754.635 & 807.058 & 23.020 & 23.807 & 93.5 & 45.85 \\
\hline 6. & Days to maturity & $\begin{array}{l}108.00- \\
142.33\end{array}$ & $124.14 \pm 4.52$ & 37.369 & 98.692 & 4.924 & 8.002 & 37.9 & 6.24 \\
\hline 7. & $\begin{array}{l}\text { Flag Leaf area } \\
\left(\mathrm{cm}^{2}\right)\end{array}$ & $\begin{array}{l}19.32- \\
45.91\end{array}$ & $29.38 \pm 1.28$ & 38.992 & 43.976 & 21.249 & 22.566 & 88.7 & 41.21 \\
\hline 8. & Filled & $69.36-$ & $151.51 \pm 5.71$ & 483.139 & 581.173 & 19.028 & 20.869 & 83.1 & 35.73 \\
\hline
\end{tabular}




\begin{tabular}{|c|c|c|c|c|c|c|c|c|c|}
\hline & grains/panicle & 179.23 & & & & & & & \\
\hline 9. & $\begin{array}{l}\text { Unfilled } \\
\text { grains/panicle }\end{array}$ & $4.23-77.30$ & $23.01 \pm 2.23$ & 131.389 & 147.579 & 49.802 & 52.782 & 89.0 & 96.80 \\
\hline 10. & Test weight (gm) & $\begin{array}{l}12.46- \\
28.53\end{array}$ & $20.05 \pm 0.62$ & 14.339 & $15 \cdot 522$ & 18.878 & 19.641 & 92.4 & $37 \cdot 37$ \\
\hline 11. & $\begin{array}{l}\text { Grain yield/plant } \\
\text { (g) }\end{array}$ & $4.49-30.18$ & $12.59 \pm 0.972$ & 39.295 & 42.132 & 52.465 & $54 \cdot 326$ & 93.3 & 99.05 \\
\hline 12. & $\begin{array}{l}\text { Grain yield/plot } \\
\text { (kg) }\end{array}$ & $\begin{array}{l}0.357- \\
1.418\end{array}$ & $0.754 \pm 0.062$ & 0.065 & 0.076 & 33.723 & 36.612 & 84.8 & 63.98 \\
\hline 13. & $\begin{array}{l}\text { Straw yield/plot } \\
(\mathrm{kg})\end{array}$ & $\begin{array}{l}0.598- \\
2.724 \\
\end{array}$ & $1.391 \pm 0.067$ & 0.209 & 0.222 & 33.893 & 33.880 & 93.9 & 65.56 \\
\hline
\end{tabular}

Table 8: Estimates of variability, broad sense heritability and genetic advance for quality characters of rice grain

\begin{tabular}{|c|c|c|c|c|c|c|c|c|c|}
\hline $\begin{array}{l}\text { S. } \\
\text { No. }\end{array}$ & Characteristics & Range & $\operatorname{mean} \pm \mathrm{SE}$ & $\sigma^{2} \mathrm{~g}$ & $\sigma^{2} p$ & GCV & $\mathbf{P C V}$ & $\begin{array}{l}h^{2} \text { (broad } \\
\text { sense) }\end{array}$ & $\begin{array}{l}\text { G. A. as \% of } \\
\text { mean (5\%) }\end{array}$ \\
\hline 1. & $\begin{array}{l}\text { Length breadth } \\
\text { ratio }\end{array}$ & $\begin{array}{l}2.39- \\
4.61\end{array}$ & $3.08 \pm 0.088$ & 0.343 & 0.36 & 18.700 & 19.32 & 93.6 & 37.27 \\
\hline 2. & Elongation ratio & $\begin{array}{l}1.09- \\
1.68\end{array}$ & $1.27 \pm 0.033$ & 0.018 & 0.021 & 10.484 & 11.45 & 83.7 & 19.76 \\
\hline 3 . & Elongation index & $\begin{array}{l}0.75^{-} \\
1.09\end{array}$ & $0.92 \pm 0.046$ & 0.004 & 0.01 & 6.96 & 11.11 & 39.2 & 8.98 \\
\hline 4. & $\begin{array}{l}\text { Volume } \\
\text { expansion }\end{array}$ & $\begin{array}{l}0.33^{-} \\
3.00\end{array}$ & $1.69 \pm 0.078$ & 0.530 & 0.54 & 43.011 & 43.73 & 96.7 & 87.12 \\
\hline 5 . & Water absorption & $\begin{array}{l}0.52- \\
2.91\end{array}$ & $1.67 \pm 0.078$ & 0.336 & 0.35 & $34 \cdot 573$ & 35.49 & 94.9 & 69.36 \\
\hline 6. & $\begin{array}{l}\text { Relative density } \\
(\mathrm{g} / \mathrm{ml})\end{array}$ & $0.57-1.87$ & $1.18 \pm 0.051$ & 0.078 & 0.086 & 23.469 & 24.64 & 90.7 & 46.04 \\
\hline 7. & $\begin{array}{l}\text { Protein content } \\
\text { (\%) }\end{array}$ & $\begin{array}{l}6.54^{-} \\
11.82\end{array}$ & $8.70 \pm 0.451$ & 2.317 & 2.92 & 17.491 & 19.66 & 79.1 & 32.05 \\
\hline 8. & $\begin{array}{l}\text { Gel consistency } \\
(\mathrm{mm})\end{array}$ & $\begin{array}{l}24.33^{-} \\
96.33\end{array}$ & $64.72 \pm 1.84$ & 220.017 & 230.20 & 22.918 & 23.44 & 95.6 & 46.15 \\
\hline 9. & $\begin{array}{l}\text { Amylose content } \\
\text { (\%) }\end{array}$ & $\begin{array}{l}18.53^{-} \\
32.2^{-}\end{array}$ & $24.80 \pm 1.01$ & 12.767 & 15.03 & 14.405 & 15.63 & 84.9 & $27 \cdot 34$ \\
\hline
\end{tabular}

\section{Heritability and genetic advance}

Heritability in broad sense estimates were highest for straw yield per plot (93.9\%) followed by number of effective tillers per plant (93.5\%),grain yield per plant (93.3\%), 1000 grains weight (92.4\%), number of effective tillers per plant (91.7\%), number of unfilled grains per panicle (89.0\%), flag leaf area (88.7\%), grain yield per plot $(84.8 \%)$, number of filled grains per panicle $(83.1 \%)$ and plant height (78.2\%) among yield contributing characters, whereas, among grain quality characters volume expansion (96.7\%) had recorded highest estimate of broad sense heritability followed by gel consistency (95.6\%), water absorption per unit rice (94.9\%), L: B ratio (93.6\%), relative density (90.7\%), amylose content (84.9\%) and elongation ratio $(79.1 \%)$. The estimates of heritability were moderate for days to 50\% flowering (43.9\%) followed by elongation index (39.2\%), panicle length (39.0\%) and days to maturity $(37.9 \%)$.

Similarly, genetic advance as percent of mean were recorded for grain yield and quality traits. Among yield contributing characters, the high expected genetic advance were observed for the characters viz., grain yield per plant (99.05\%), number of unfilled grains per panicle (96.80\%), straw yield per plot $(65.56 \%)$, grain yield per plot (63.98\%), number of effective tillers per plant (52.89\%), number of effective tillers per meter length (45.85\%), flag leaf area $(41.21 \%)$, test weight $(37.37 \%)$, number of filled grains per panicle (35.73\%) and plant height (34.54\%).
However, lowest expected genetic advance was observed for days to maturity (6.24\%), panicle length (7.62\%) and days to $50 \%$ flowering (8.64\%). Among the quality characters, moderate expected genetic advance was observed in elongation ratio (19.76\%) and lowest in elongation index (8.98\%).

Among the grain yield contributing characters, high estimates of GCV and heritability for grain yield per plant, number of effective tillers per plant and water absorption was observed. Similar, results were also reported earlier by Durai et al. [40] and Chaudhari et al. [41]. Parameters showed high estimates of PCV, high heritability with high genetic advance as percent of mean, indicated that role of additive gene effects in the inheritance of traits, these results are in conformity with the findings of Govindswami and Ghosh [42], Kumari et al. [43] and Selvaraj et al. [44]. Fukrei et al. [28] observed high PCV, high heritability and high genetic advance as percent of mean for flag leaf area, straw yield and gain yield while Bisne et al. [45] observed similar result for number of unfilled grains per panicle. Low genetic advance as percent of mean was recorded by panicle length, days to 50\% flowering and days to maturity coupled with moderate heritability. Similar low genetic advance as percent of mean was noted by Babu et al. [46], Prajapati et al. [26] and Singh et al. [40] for panicle length; Pandey et al. [47] observed for days to 50\% flowering, and days to \% flowering and days to maturity. 
Grain elongation without increase in girth is considered highly desirable in some high quality rice. Basmati rice elongates 100 per cent upon cooking. In the present study elongation ratio exhibited high heritability coupled with moderate EGA which was accordance with the findings of Vanaja and Babu [48] and Subbaiah et al. [30]. Likewise, gel consistency measures the tendency of the gelatinized starch to retrograde on cooking, varieties with soft gel consistency are preferred. High heritability with high EGA was observed for gel consistency indicating that character is controlled by additive gene action and thus selection would be effective for that character. Similar result was also reported by Babu et al. [19].

The high heritability coupled with high EGA was observed for water absorption, amylose content, relative density, protein content, volume expansion and L: B ratio. Similar findings were also reported by Chouhan and Nanda [49], Hussain et al. [50] and Nayak et al. [51] for water absorption and amylose content while Babu et al. [19] observed for L: B ratio and volume expansion ratio. Vanaja and Babu [48] observed similar results for L: B ratio, volume expansion, water uptake and amylose content. High heritability and high EGA was recorded for protein content, which was in contrary with the result observed by Shenoy et al. [52].

\section{CONCLUSION}

It is presumed that dwarf, high yielding and slender grain rice having quality traits viz., high protein, intermediate amylose and soft gel consistency are the characteristics of rice preferred by people in Maharashtra. The present investigation revealed that these genotypes possess better grain and cooking quality characters including good agronomic traits. The semi dwarf genotypes Tulasi, NDR 1143, CRR 599-4-1, PBNR 04-28, CRR 3630-1-3, CRR 6614-1-4, PBNR 0807 and PBNR 03-10 possess maximum number of good attributes and higher grain yield per plant. These genotypes need to be improving for its cooking quality characters. Tall genotypes PBNR 03-07 seemed to be promising but need improvement in some of the quality characters. The genotype NDR 1143 had more yield and protein content than the checks and also possess some good quality characters. The genotypes PAU 3832-79-4$3^{-1}(11.82 \%)$ and UPRI (11.59\%) recorded the highest protein content. The promising genotype for yield and grain cooking quality characters requires further study for its confirmation. In the present investigation, appreciable amount of GCV, heritability and EGA was observed for quantitative characters. The characters that showed high heritability coupled with high genetic advance are controlled by additive gene action which is coherent with the findings of Panse and Sukhatme [15]. Similarly, for the characters having high heritability along with moderate and low genetic advance has possibilities for improving by inter-mating with superior genotypes of segregating population.

\section{ACKNOWLEDGEMENT}

The authors would like to acknowledge faculty members of Department of Agricultural Botany, VNMKV; Vijaya Boda, Rajdeep Bansode, Pritesh Tyade and all the support staffs of Department of Genetics and Plant Breeding of VNMKV for technical support in conducting this research.

\section{AUTHORS CONTRIBUTIONS}

Rajeev Dhakal is the principal researcher who conducted the entire experiment and meticulously recorded the data. Ritesh Kumar Yadav helped the principal author in statistical analyses and interpretation of the results, and drafting this manuscript. Dr. D. B. Deosarkar is Professor, Department of Agricultural Botany, VNMKV who conceptualized, designed and supervised the research. D. P. Paudel guided the principal researcher during various stage of research and provided his critical suggestions and feedbacks for refinement of this manuscript.

\section{REFERENCES}

1. Bucheyeki LT, Iwa SE, Lobulu J. Assessment of rice production constraints and farmers preferences in Neega and Igunga districts. J. Adv. Dev. Res. 2011;2(1).

2. Food and Agriculture Organization (FAO). Rice Information. 2000. Vol. 22. January. 2000

3. Sreedhar S, Vanisree S, Kulakarni N, Ganesh M. Gene effects for certain physical quality traits and grain yield in rice. Madras Agricultural Journal. 2005;92:183-187.

4. Anonymous. Progress report, Kharif. $48^{\text {th }}$ Annual Maharashtra state rice workshop. 2014.

5. De Kock PC. Fundamental aspects of iron nutrition of plants. In: Proceedings of Conf. of Traces Elements in Soils and Crops. London.1966. Pp 41-44. H M 50, London; 1971.

6. Hoan NT, Prasada Rao U, Siddiq EA. Genetics of tolerance to iron chlorosis on rice. Plant and Soil. 1992;146:233-239.

7. Deosarkar DB. Inheritance of iron chlorosis tolerance in vertisols, plant height, yield and grain quality traits in indica rice (Oryza sativa L.). $\mathrm{PhD}$ Thesis. Marathwada Agricultural University. 1989.

8. Dhakal R, Deosarkar DB, Paudel DP. Studies of correlation and path analysis of grain yield with yield contributing characters, grain quality characters and iron chlorosis in aerobic (Oryza sativa L.) on Vertisols. International Journal of Current Research. 2017;9:52926-52931.

9. Singh BN, Singh BP. Tolerance for iron chlorosis in rice. Int. Rice Res. Newslett. 1984;2(6):8-9.

10. Jadhav AS, Dahiphale VV, Gunjkar MU. Leaf area constant for basmati and non-basmati cultivars of rice under upland condition. Annals of Plant Physiology. 2001;14(1):5-6.

11. Parikh M, Rastogi NK, Sarawgi AK. Variability in grain quality traits of aromatic rice (Oryza sativa L.). Bangladesh J. Agril. Res. 2012;37(4):551-558.

12. AOAC. Official methods of analysis. Association of Official Analytical Chemists, $10^{\text {th }}$ edn. Washington, D. C. 1965 .

13. Cagampang GB, Perez CM, Juliano BO. A gel consistency test for eating quality of rice. J. Sci. Food Agr. 1973;24:1589-1594.

14. Juliano BO. A simplified assay for milled rice amylose. Cereal Science Today. 1971;16:334-340.

15. Panse VG, Sukhatme PV. Genetics and quantitative characters in relation to plant breeding. Indian $\mathrm{J}$. Genetics. 1957;17:312-328.

16. Burton GW. Quantitative inheritance in grasses. Proc. Int. Grassland Congress. 1952;1:277-283.

17. Hanson $\mathrm{CH}$, Robinson HF, Comstock RE. Biometrical studies of yield in segregating population of Korean Lespedeza. Agronomy Journal. 1956;48:268-272. 
18. Johnson HW, Robinson HF, Comstock RE. Estimation of genetic and environmental variability in soybean. Agronomy Jounal.1955;47:314-318.

19. Babu VR, Shreya K, Dangi KS, Usharani G, Nagesh P. Genetic variability studies for qualitative and quantitative traits in popular rice (Oryza sativa L.) hybrids of India. International J. of Scientific and Research Publications 2012;2(4):1-4.

20. Dhanwani RK, Sarawgi AK, Solanki A, Tiwari JK. Genetic variability analysis for various yield attributing and quality traits in rice (Oryza sativa L.). The Bioscan. 2013;8(4):1403-1407.

21. Shejul MB, Deosarkar DB, Kalpande HV, Chavan SK, Deshmukh VD, Dey U, Kumar A, Bhandhavi RD, Arbad SK. Variability studies for grain quality characters in upland rice (Oryza sativa L.). African Journal of Agricultural Research. 2013;8(38):4872-4875.

22. Das PK, Miah NM, Loresto GC. Genetic variability in root and shoot character of selected rice genotypes. Int. Rice Res. Newslett. 1989;16 (5):65-67.

23. Binod KA, Kalayarasi R, Thiyagarajan K, Manomani S. Physico-chemical and cooking quality characteristics of promising varieties and hybrids in rice (Oryza sativa L.). Indian J. Genet. 2006;66 (2):107-112.

24. Panwar LL, Mathur SS. Variability studies in segregating populations of Rice (Oryza sativa L.). Ann. Agric. Res. New Series. 2007;28(1):53-56.

25. Devi SL, Raina FA, Pandey MK, Cole T. Genetic parameters of variation for yield and its components in rice. Crop Res. 2006;32 (1):69-71.

26. Prajapati M, Singh CM, Suresh BG, Lavanya GR, Jadhav P. Genetic parameters for grain yield and its component characters in rice. Elec. J. Pl. Breeding. 2011;2(2): 235-238.

27. Tuwar AK, Singh SK, Sharma A, Bhati PK. Appraisal of genetic variability for yield and its component characters in rice (Oryza sativa L.). Biolife: 2013;1(3): 84-89.

28. Fukeri KP, Kumar A, Tyagi W, Rai M, Pattanayak A. Genetic variability in yield and its components in upland rice grown in acidic soils of north east India. J. of Rice Res. 2011;4(1):4-7.

29. Subbaiah PV, Sekhar MR, Reddy KHP, Eswara Reddy NP. Variability and genetic parameters for grain yield and its components and kernel quality attributes in CMS based rice hybrids (Oryza sativa L.). International Journal of Applied Biology and Pharmaceuticals Technology. 2011;2(3):603-609.

30. Kundu A, Senapati BK, Bakshi A, Mandal GS. Genetic variability for panicle characters in tall indica aman rice. Oryza. 2008;45(4):320-323.

31. Shukla TC, Kandekar MP, Sahu RK. Phenotypic variation and its heritable components in quantitative yield contributing characters in rice (Oryza sativa L.) Indian J. Agric. Sci. 1972;42(8):660-663.

32. Kaul ML, Kumar V. Genetic variability in rice. Genetica Agraria. 1982;36(4):257-268.

33. Debchoudhary PK, Das PK. Genetic variability, correlation and path coefficient analysis in deep water rice. Annals of Agricultural Research. 1998;19:120-124.

34. Chauhan JS. Segregation analysis and estimation of genetic parameters for some quality traits in $F_{2}$ generation in rice. Oryza. 1996;33:168-173.

35. Shivani D, Reddy NSR. Variability, heritability and genetic advance for morphological and physiological traits in certain rice hybrids. Oryza. 2000;37(3): 231-233.
36. Anandrao SD, Singh CM, Suresh BG, Lavanya GR. Evaluation of rice hybrids for yield and yield component characters under North East Plain Zone. The Allahabad Farmer. 2011;67(1): 63-68.

37. Singh SK, Singh CM and Lal GM. Assessment of genetic variability for yield and its components characters in rice (Oryza sativa L.). Research in Plant Biology. 2011;1(4):73-76.

38. Sinha SK, Tripathi AK, Bisen UK. Study of genetic variability and correlation co-efficient analysis in mid land landraces of rice. Ann. Agric. Res. New Series. 2004;25:1-3

39. Sadeghi SM. Heritability, phenotypic correlation and path coefficient studies for some agronomic characters in landraces of rice varieties. World Applied Sciences Journal. 2011;13(5):1229-1233.

40. Durai AA, Ngachan SV, Pattanayak A, Sarma BK. Comparative study of heritability, genetic advance and association of characters in conventionally bred and doubled haploid lines of rice (Oryza sativa L.). Indian Journal of Hill Farming. 2001;14(2):71-75.

41. Chaudhari M, Motirmani NK, Pravin J. Variability and genetic divergence of aromatic rice. (Oryza sativa L.). Ann. Agric. Res. New series. 2007;28:268-272.

42. Govindswami S, Ghosh AK. Genetic variability and correlation studies on protein content and some quantitative characters of rice (Oryza sativa L.), Oryza. 1973;10(1):1-8.

43. Kumari RU, Rangasamy P, Gomez SM. Phenotypic differentiation in indica-japonica wide compatible varieties in rice (Oryza sativa L). Plant Archives. 2003;3(1):141-142.

44. Selvaraj CI, Pothiraj N, Thiyagarajan K, Bharathi M, Rabindran R. Genetic parameters of variability, correlation and path-coefficient studies for grain yield and other yield attributes among rice blast disease resistant genotypes of rice (Oryza sativa L.). African J. of Biotechnology. 2011;10 (17):3322-3334.

45. Bisne R, Sarawgi AK, Verulkar SB. Study of heritability, genetic advance and variability for yield and yield contributing characters in rice. Bangladesh J. Agril. Res. 2009;34(2):175-179.

46. Babu SG, Lavanya, Singh AP. Genetic variability for grain yield and character association studies in upland rice (Oryza sativa L.). Envir. Ecol. 2011;29 (1):164168.

47. Pandey P, Anurag PJ, Rangare NR. Genetic parameters for yield and associated characters in rice. Ann. Pl. Soil. Res. 2010;12(1):59-61.

48. Vanaja T, Babu LC. Variability in grain quality attributes of high yielding rice varieties (Oryza sativa L.) of diverse origin. Journal of Tropical Agriculture. 2006;44:61-63.

49. Chauhan JS, Nanda JS. Genetic variability for physicchemical characters of rice grain in segregating Oryza sativa L. Oryza. 1983;20:209-215.

50. Hussain AA, Maurya DM, Vaish CP. Studies on quality status of indigenous upland rice (O. Sativa L.). Indian J. Genet. 1987;47(2):145-151.

51. Nayak AR, Chaudhary D, Reddy JN. Genetic variability and correlation study among quality characters in scented rice. Agric. Sci. Digest. 2003;23(3):175-178.

52. Shenoy VV, Seshu DV, Sachan JKS. Inheritance of protein per grain in rice. Indian. J. Genet. 1991;51:214-220. 